\title{
First results from the multichannel interferometer system on HSX
}

\author{
C. Deng, ${ }^{\text {a) }}$ D. L. Brower, and W. X. Ding \\ Electrical Engineering Department, University of California, Los Angeles, Los Angeles, California
}

\author{
A. F. Almagri, D. T. Anderson, F. S. B. Anderson, S. P. Gerhardt, P. Probert, \\ and J. N. Talmadge \\ The HSX Plasma Laboratory, University of Wisconsin, Madison, Wisconsin
}

(Presented on 8 July 2002)

\begin{abstract}
Measuring the equilibrium electron density distribution and its response to perturbations provides important information to magnetically confined plasma research. A multichannel interferometer system is now routinely operating on the new quasihelically symmetric stellator (HSX) to measure the equilibrium profile and electron density dynamics. The interferometer system has nine viewing chords with $1.5 \mathrm{~cm}$ spacing. The source is a bias-tuned Gunn diode at $96 \mathrm{GHz}$ with passive solid-state tripler providing output at $288 \mathrm{GHz}(8 \mathrm{~mW})$. The HSX plasma is produced by $28 \mathrm{GHz}$ electron cyclotron resonance heating and first results of the interferometer measurement are reported. The density spatial distribution is reconstructed from the measured line-integrated density. At high density $\left[\bar{n}_{e}>2 \times 10^{12} \mathrm{~cm}^{-3}\right]$, an $m=1$ density oscillation with frequency of $1-2 \mathrm{kHz}$ is observed. Plans to determine the radial particle flux and transport coefficients will be discussed.
\end{abstract} (C) 2003 American Institute of Physics. [DOI: 10.1063/1.1530353]

\section{INTRODUCTION}

A new concept stellarator, the helically symmetric experiment (HSX), has been built at the University of Wisconsin-Madison. ${ }^{1}$ HSX was designed to have virtually no toroidal curvature and a helical axis of symmetry which eliminates the fundamental disadvantage of conventional stellarators: poor neoclassical transport in the low collisionality regime. The quasihelically symmetric (QHS) magnetic field configuration of HSX is produced by modular coils. In addition, HSX has a set of auxiliary coils that allow the magnetic topology to be altered. In the mirror mode configuration, the helical symmetry is broken by making the field mirror-like along the magnetic axis. In this case, the neoclassical transport is similar to that in a conventional stellarator. For the anti-mirror mode configuration, the mirror phase is reversed, but the global transport remains unchanged. Understanding plasma confinement and particle transport in a stellarator with and without a helical axis of symmetry requires information on both the electron density distribution as well as density fluctuations.

\section{HSX INTERFEROMETER SYSTEM}

To attain the physics goals of HSX, a high-resolution interferometer system has recently been installed. ${ }^{2}$ This system has nine evenly distributed spatial channels with $1.5 \mathrm{~cm}$ spacing and width. The interferometer is designed to measure the plasma density distribution at one of the four box ports where the closed flux surfaces are highly elongated but symmetric about the midplane. The nine interferometer channels cover the plasma cross section from the outboard side scrape-off layer to well past the magnetic axis. The last

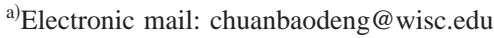

closed flux surface for the QHS operational mode and the location of the nine interferometer chords are shown in Fig. 1. Details of the optical system, detection scheme, and system calibration have been previously described elsewhere ${ }^{2}$ and will only be briefly reviewed here.

Radiation used for both the probe and local-oscillator beams is generated by a solid-state source operating at 288 GHz. The source consists of a $96 \mathrm{GHz}$ bias-tuned Gunn oscillator and an InP tripler which generates up to $8 \mathrm{~mW}$ of output power at $288 \mathrm{GHz}$. Frequency modulation at $775 \mathrm{kHz}$ (intermediate frequency) is achieved by sweeping the voltage of the Gunn oscillator. Quasioptical techniques are used for

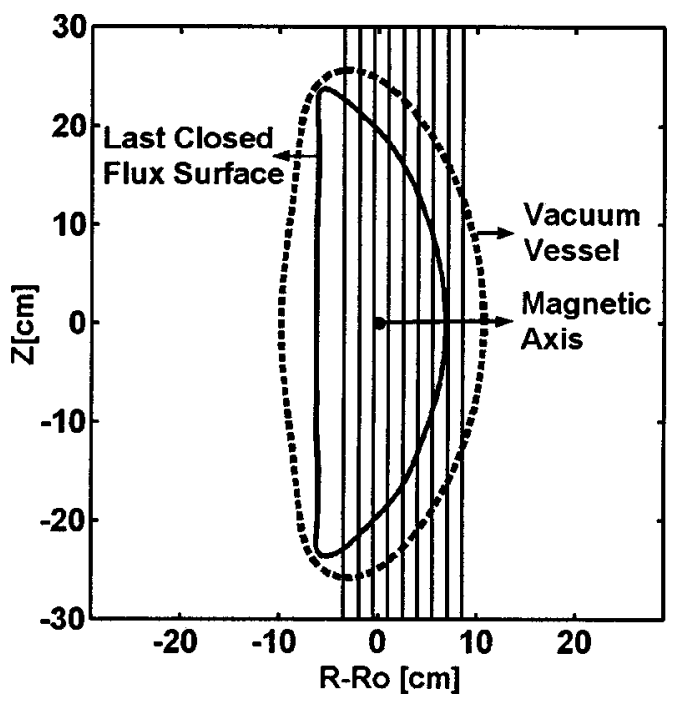

FIG. 1. Schematic of HSX cross section (at box port) showing the last closed magnetic flux surface for QHS mode and position of the nine interferometer chords. The magnetic axis corresponds to $R-R_{0}=0$; the last closed flux surface is located at $x=6.8 \mathrm{~cm}$ on the outboard side. 
propagation of the beams. A multi-chord system is realized by beam expansion where parabolic cylindrical mirrors are used to match the beam size to the port dimensions $(13.5 \mathrm{~cm}$ $\times 3.25 \mathrm{~cm}$ ). Detection is accomplished by a mixer array of nine corner-cube GaAs Schottky diodes. Dichroic filters (cutoff $\sim 220 \mathrm{GHz}$ ) are mounted on the entrance and exit ports of the HSX vacuum vessel to protect the source and mixers from stray $28 \mathrm{GHz}$ gyrotron radiation and its harmonics.

The interferometer phase is measured in two ways. First, analog phase detection electronics (APC) with bandwidth $\sim 10 \mathrm{kHz}$ are used to provide real-time output from each channel. These data can be used to determine the equilibrium profile and its evolution. A digital phase detection technique $(\mathrm{DPC})^{3}$ is also employed to provide phase information with time response up to $\sim 200 \mathrm{kHz}$. System noise on the lineintegrated density trace from the analog phase comparator is about $1.6^{\circ}$, corresponding to $n_{e} d l \sim 0.9 \times 10^{12} \mathrm{~cm}^{-2}$. For digital phase detection with $200 \mathrm{kHz}$ bandwidth, the noise level is $n_{e} d l \sim 1.5 \times 10^{12} \mathrm{~cm}^{-2}$.

\section{EXPERIMENTAL RESULTS}

HSX plasmas are produced and heated by electron cyclotron resonance heating (ECRH) using up to $200 \mathrm{~kW}$ of 28 $\mathrm{GHz}$ gyrotron radiation. Results presented here are for launched power $\leqslant 50 \mathrm{~kW}$. Typical line-integrated densities from the nine viewing chords measured by the interferometer are shown in Figs. 2(a) and 2(b). The ECRH pulse starts at $800 \mathrm{~ms}$ and lasts approximately $25 \mathrm{~ms}$. For these data, the magnetic configuration is QHS mode with the ECRH resonant position located at the magnetic axis $\left(R-R_{0}=0 \mathrm{~cm}\right)$. After an initial startup phase (800-808 ms), the density levels off with peak density $n_{e} d l \sim 4 \times 10^{13} \mathrm{~cm}^{-2}$. The $500 \mathrm{~Hz}$ oscillations during the flattop period (808-825 ms) are produced by modulating the gas feed. After the ECRH turn-off $(825 \mathrm{~ms})$, the central density increases up to $50 \%$ within 2.5 $\mathrm{ms}$ and then gradually decays. However, the plasma stored energy and the $H_{\alpha}$ signal decay to zero within 1-2 ms. The approximate total number of electrons can be obtained from integration of chord averaged data and is observed to increase $\sim 40 \%$ after ECRH turn-off as shown in Fig. 2(c). The density rise after ECRH turn-off may be related to the slowing down of the energetic electrons. Experimentally, the density rise after ECRH is observed for all the three magnetic configurations (QHS, mirror and antimirror modes) providing the vacuum vessel walls are clean.

The line-integrated density determined by using the two independent phase analysis techniques, APC and DPC, are found to be in good agreement as shown in Fig. 3. APC provides a real-time monitoring of the electron density for each channel with bandwidth less than $10 \mathrm{kHz}$. For the DPC approach, each signal wave form is sampled at $1 \mathrm{MHz}$ causing the $775 \mathrm{kHz}$ IF frequency to be aliased to $225 \mathrm{kHz}$. This limits the total system bandwidth to $<225 \mathrm{kHz}$. The digital phase comparator approach allows measurement of fast dynamic changes to the equilibrium profile as well as electron density fluctuations. Both phase measurement techniques are routinely used on HSX.

The electron density profile is obtained from the mea-

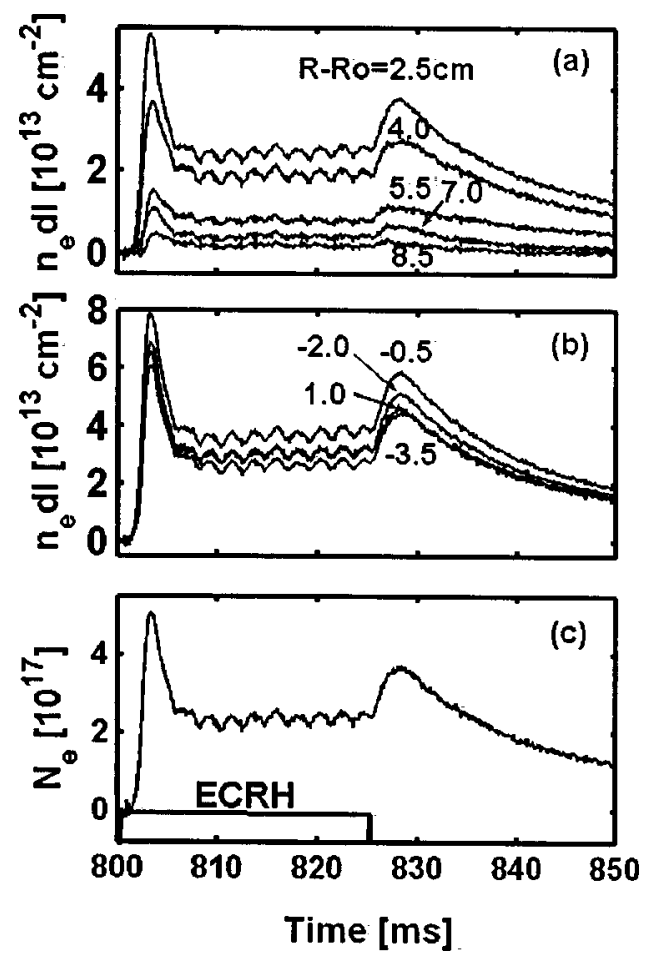

FIG. 2. (a), (b) Line-integrated density time traces from digital phase comparator; (c) total number of electrons for a QHS discharge with $50 \mathrm{~kW}$ ECRH from 800 to $825 \mathrm{~ms}$.

sured line-integrated density by using an Abel inversion technique described by Park. ${ }^{4}$ On HSX, the flux surface geometry is determined by external coils where the Shafranov shift can be ignored for present $\beta$ values. The plasma phase shift is

$$
\Phi=\frac{\lambda e^{2}}{4 \pi c^{2} \varepsilon_{0} m_{e}} \int n_{e} d z=2.82 \times 10^{-15} \lambda \int n_{e} d z,
$$

where $\lambda$ is the wavelength of probing radiation. If constant density is assumed on a flux surface, the line integrals can be represented as $\Phi_{i}=c \sum_{j=1}^{m} L_{i j} n_{j}$, where $c$ is a constant, $\Phi_{i}$ is

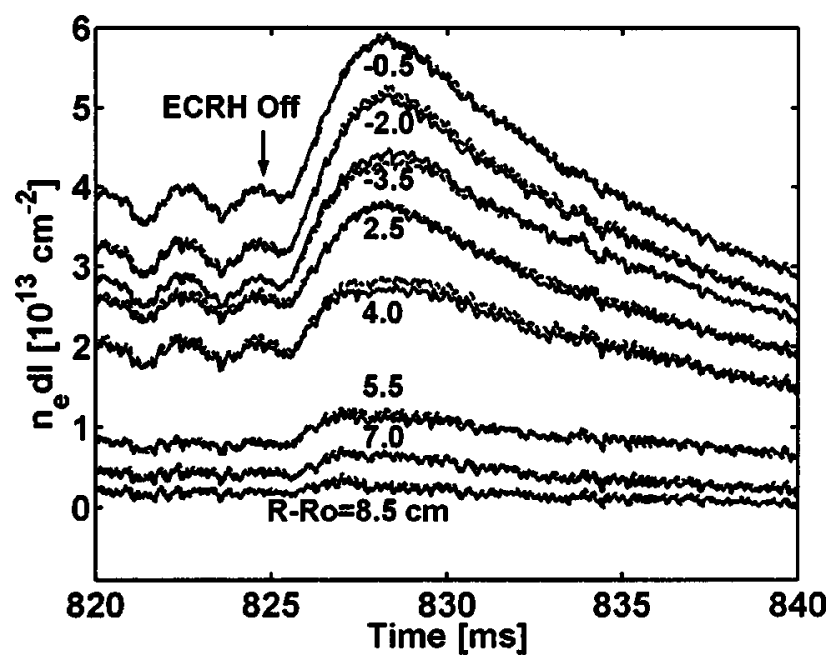

FIG. 3. Comparison between analog (dashed line) and digital (solid line) phase detection techniques. 

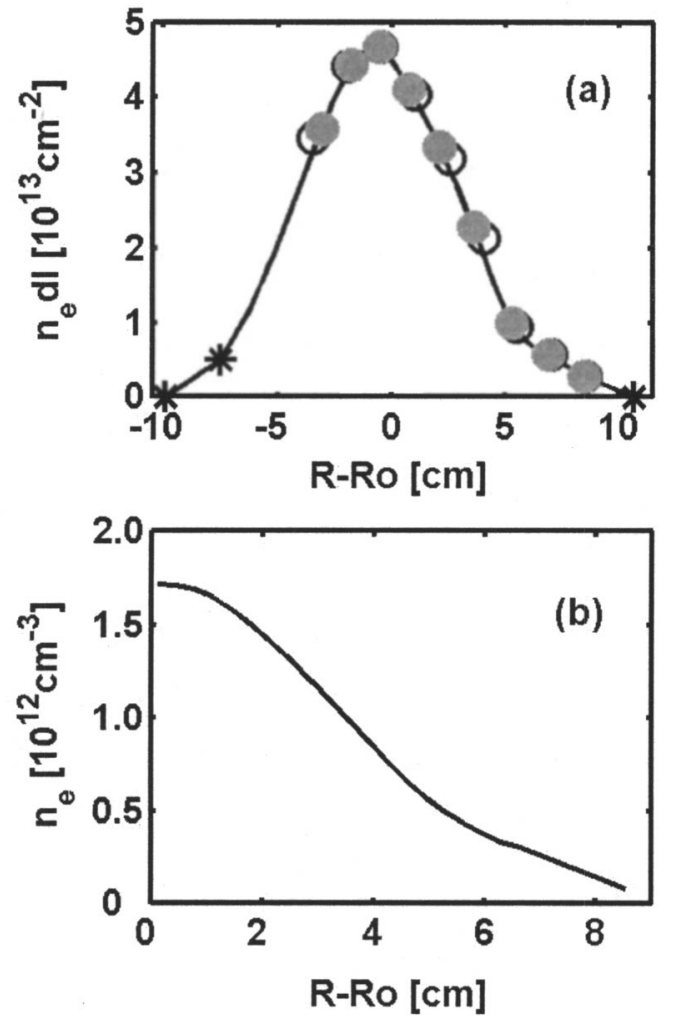

FIG. 4. (a) Line-integrated density profile for a QHS mode discharge, where open circles are measured data, solid circles are data after refraction correction, stars are extrapolated data, and the solid line is a spline fit to the points after refraction correction. (b) Inverted density profile.

the measured phase change for a given chord, $n_{j}$ is the density on the flux surface, $m$ is the number of flux surfaces, and $L_{i j}$ is the path length matrix. Solving the linear equation for density using the singular value decomposition $\operatorname{method}^{5}$ gives the density profile as shown in Fig. 4. Refraction of the probe beam due to the plasma density gradient causes changes to both the viewing position and chord length. These effects are accounted for when performing the inversion and are only significant for densities $>1 \times 10^{12} \mathrm{~cm}^{-3}$. The HSX density profile is strongly peaked on axis for the QHS configuration.

The line-integrated electron density profiles vary for the three different magnetic configurations (QHS, mirror, antimirror) as shown in Fig. 5. For each magnetic configuration, the position of the magnetic axis changes. These shifts can be seen in the profile data. Calculations indicate that the magnetic axis shifts $\sim 1 \mathrm{~cm}$ (out) in for the (anti-) mirror mode. The flux surface shape and chord lengths for the QHS configuration have been calculated for the density profile inversion. However, the neural net code used to calculate the flux surface geometry for the mirror mode configurations has not been completed, thereby preventing inversion of the lineintegrated profiles.

The interferometer is capable of measuring highfrequency density perturbations due to magnetohydrodynamic behavior or turbulence $\left(k_{\max }=2.1 \mathrm{~cm}^{-1}\right) .^{2}$ On HSX, when the line-averaged density is above $2 \times 10^{12} \mathrm{~cm}^{-3}, 1-2$ $\mathrm{kHz}$ oscillations with $m=1$ mode characteristics are often

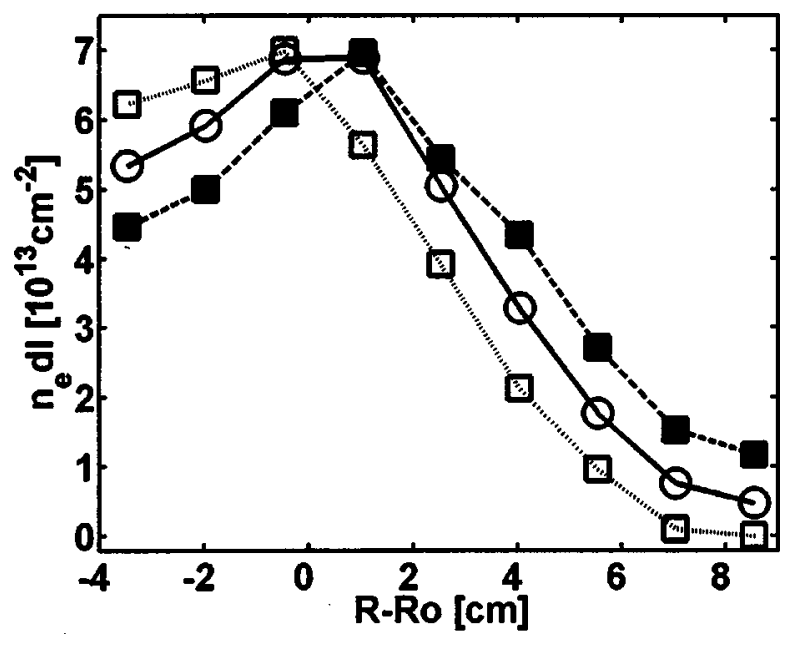

FIG. 5. Comparison of line-integrated density profiles for the three magnetic configurations where open circles correspond to QHS mode, open squares correspond to Mirror mode, and solid squares correspond to antimirror mode.

observed as shown in Fig. 6. One can clearly see that the density oscillations for channels positioned inside the magnetic axis are out of phase with channels outside of the magnetic axis, characteristic of an $m=1$ mode. However, for channels beyond $5.5 \mathrm{~cm}$, the oscillations are not observed, indicating the modes are spatially localized to the plasma core.

\section{PARTICLE TRANSPORT}

Measuring and understanding particle transport will be a crucial issue in determining whether the QHS configuration achieves improved performance compared to conventional stellarators. The equilibrium electron density profile, in conjunction with particle source measurements, can be used to study particle transport by providing information on the net radial particle flux. Perturbative particle transport studies have also been proven useful in determining the electron transport coefficients. ${ }^{6,7}$ On HSX, electron density perturbations can be produced by modulating the external gas feed. The perturbed line-integrated density time traces during gas modulation for a QHS discharge are shown in Fig. 7(a) The amplitude and phase of the line-integrated density perturbation versus position is plotted in Figs. 7(b) and 7(c), respectively. The observed fluctuation-amplitude asymmetry about the magnetic axis is due to the path length difference between inboard and outboard chords. The density fluctuation for the central chord $(x=1 \mathrm{~cm})$ is delayed $\sim 0.16 \mathrm{~ms}$ with respect to the edge chord $(x=7 \mathrm{~cm})$. This time scale is much less than particle confinement time $(\sim 1 \mathrm{~ms})$. However, particle flux for this QHS mode plasma is outward because of the peaked density profile. Therefore, we have to consider neutral particle transport and ionization to understand the electron transport.

Information on particle transport can be obtained by solving the continuity equation

$$
\frac{\partial n}{\partial t}=-\nabla \Gamma+S,
$$



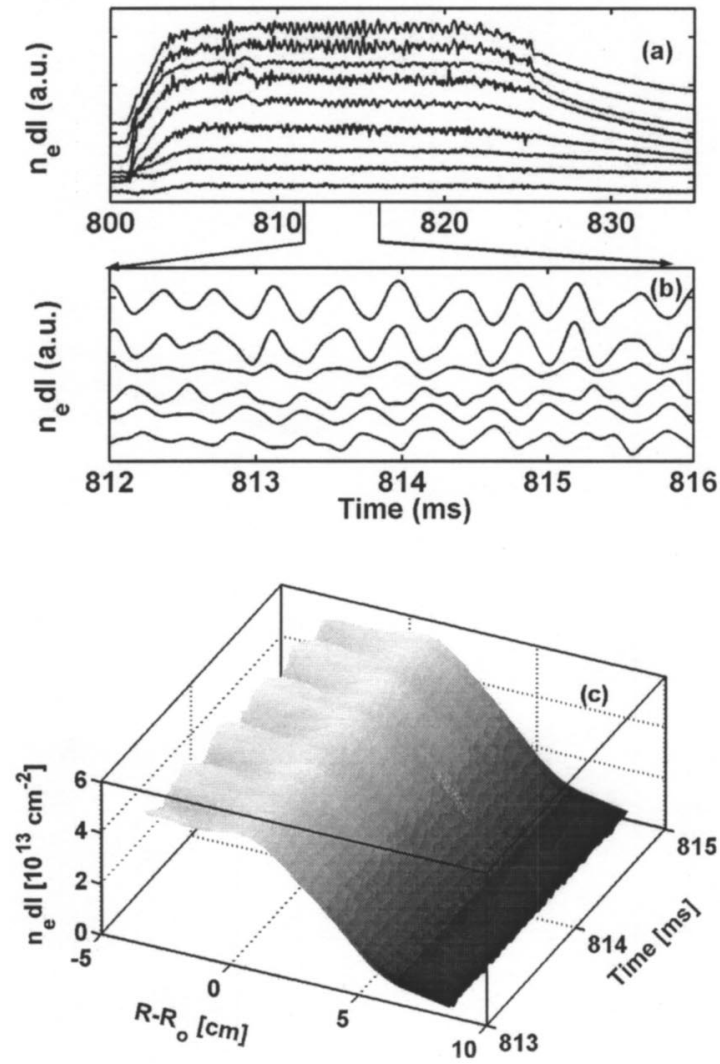

FIG. 6. (a) Line-integrated traces of $m=1$ density fluctuations at $1.5 \mathrm{kHz}$ : from bottom to top: $R-R_{0}=8.5,7.0,5.5,4.0,2.5,1.0,-0.5,-2.0,-3.5$ $\mathrm{cm}$. (b) Expanded time traces for period from 812 to $816 \mathrm{~ms}$ : from bottom to top: $R-R_{0}=4.0,2.5,1.0,-0.5,-2.0,-3.5 \mathrm{~cm}$; (c) three-dimensional plot of the line-integrated density temporal evolution.

where $\Gamma=-D \nabla n+V n . D$ is the particle diffusion coefficient, $V$ is a convection velocity, and $S$ is the particle source. The density perturbation observed in Figs. 7(b) and 7(c) can be used to model $D$ and $V$ using Eq. (2). The source term in the continuity equation must be either measured or modeled. On HSX, a ten poloidal channel and six toroidal channel $H_{\alpha}$ measurement system has been built to measure the source term for the working gas. Measurement of electron temperature is also required to calculate the electron source from the $H_{\alpha}$ emission. Information of the electron source is expected to be obtained in the near future. ${ }^{8}$

\section{CONCLUSION}

First results from the multichannel interferometer system on HSX provide a measurement of the line-integrated electron density distribution. The inverted density profile is centrally peaked for a QHS mode plasma. Density oscillations from $m=1$ like structures with frequencies of $1-3 \mathrm{kHz}$ are observed when line averaged density $\bar{n}_{e}>2 \times 10^{12} \mathrm{~cm}^{-3}$. (a)
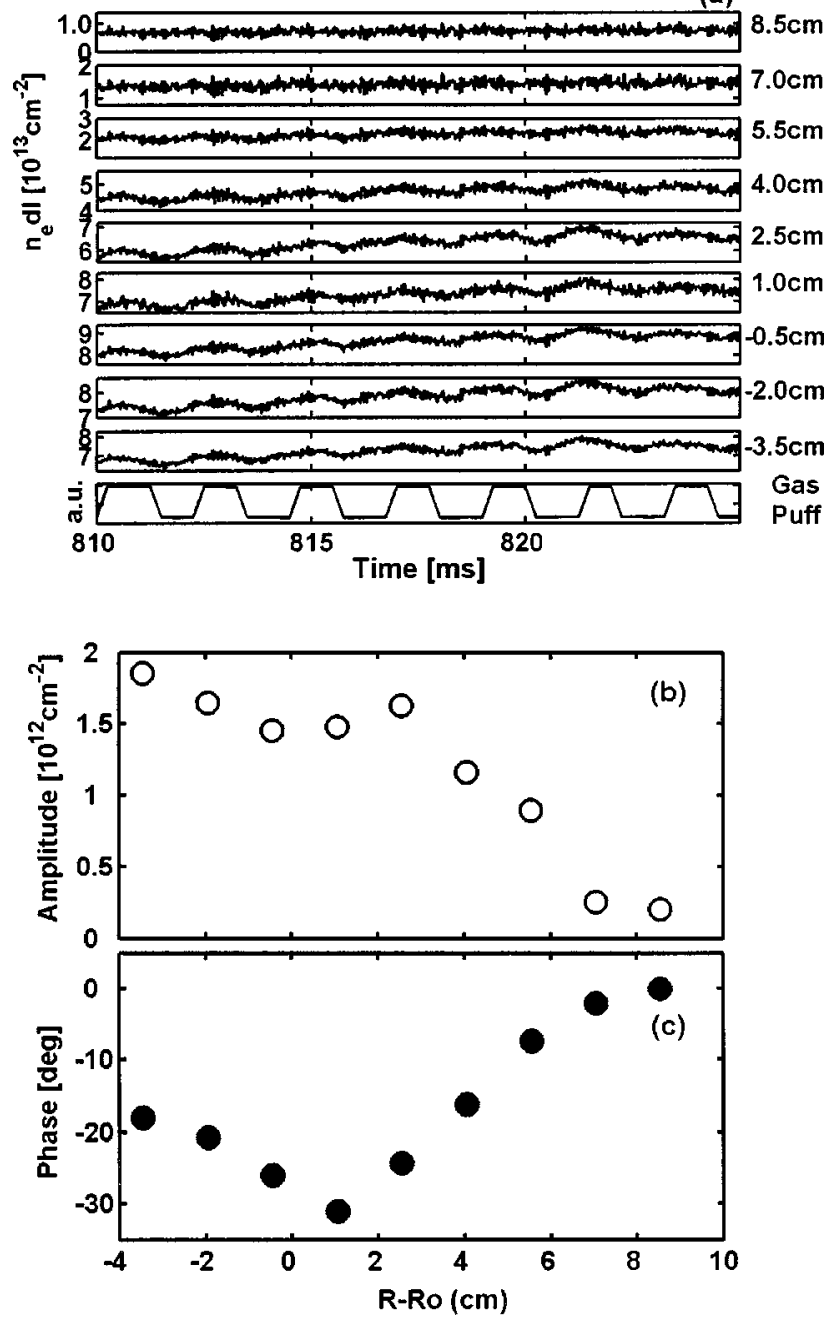

FIG. 7. (a) Line-integrated density during a gas modulation; (b) amplitude and (c) phase of the first harmonic of modulation frequency for the lineintegrated density.

The nine-channel interferometer system on HSX is used to routinely measure the electron density distribution.

\section{ACKNOWLEDGMENT}

This work is supported by the Department of Energy under Grant Nos. DE-FG03-01ER-54615, Task II and DEFG02-93ER54222.

${ }^{1}$ D. T. Anderson et al., Bull. Am. Phys. Soc. 45, 113 (2000).

${ }^{2}$ D. L. Brower, C. Deng, and W. X. Ding, Rev. Sci. Instrum. 72, 1081 (2001).

${ }^{3}$ Y. Jiang, D. L. Brower, and J. Howard, Rev. Sci. Instrum. 68, 902 (1997).

${ }^{4}$ H. K. Park, Plasma Phys. Controlled Fusion 31, 2035 (1989).

${ }^{5}$ W. H. Press, et al., Numerical Recipes in Fortran 77 and Fortran 90 (Cambridge University Press, Cambridge, 1996).

${ }^{6}$ J. P. T. Koponen, T. Geist, U. Stroth, S. Fiedler, H.-J. Hartfuss, O. Heinrich, and H. Walter, collab ECH Group, W7-AS Team, Nucl. Fusion 40, 365 (2000).

${ }^{7}$ K. W. Gentle, O. Gehre, and K. Krirger, Nucl. Fusion 32, 217 (1992).

${ }^{8}$ J. Canik, D. T. Anderson, S. P. Gerhardt, and J. N. Talmadge, Rev. Sci. Instrum. (these proceedings). 